\title{
Older Adults Designing Avatars for Socializing
}

Romina Carrasco Zuffi

Interaction Design Lab

The University of Melbourne,

Melbourne, Australia

rcarrasco@student.unimelb.edu.au

Permission to make digital or hard copies of part or all of this work for personal or classroom use is granted without fee provided that copies are not made or distributed for profit or commercial advantage and that copies bear this notice and the full citation on the first page. Copyrights for third-party components of this work must be honored. For all other uses, contact the Owner/Author.

DIS'18 Companion, June 9-13, 2018, , Hong Kong

(C) 2018 Copyright is held by the owner/author(s).

https://doi.org/10.1145/3197391.3205387

\begin{abstract}
In this research I investigate the design and use of virtual avatars (full body representations of the user) among older adults over 65 years old. This research seeks to understand the avatar's designs made by older adults in relation to the ageing body; and how the use of these self-representations impact older adults' experience when socializing online. This thesis firstly uses an exploratory approach to understand the current use of online self-representations among older adults, then design workshops to comprehend older adults' visual choices in relation to the design of humanoid avatars and finally a long-term user study where participants use and reiterate avatar designs through multiple virtual reality social sessions. This research will contribute to the understanding of how older adults use self-representations to connect with others in online environments while providing insights of how to expand character creation interfaces to cater visual preferences of those in older age.
\end{abstract}

\section{Author Keywords}

Older adults; avatars; virtual reality, self-

representation; ageing; socializing.

\section{ACM Classification Keywords}

H.1.2 [User/Machine Systems]: Human Factors. 


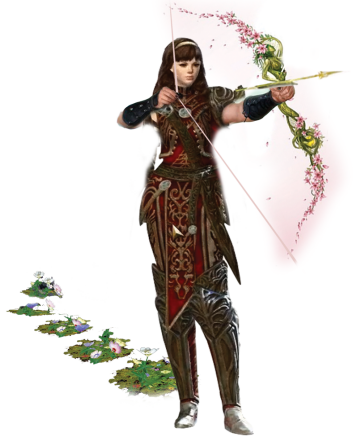

Figure 1: Hanna's (female, 68) younger self-representation in Guild Wars.

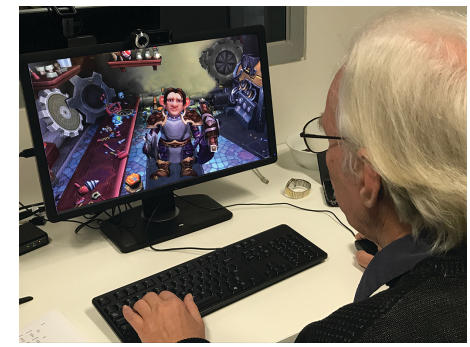

Figure 2: Jacob's (male, 66) engineer avatar in World of Warcraft.

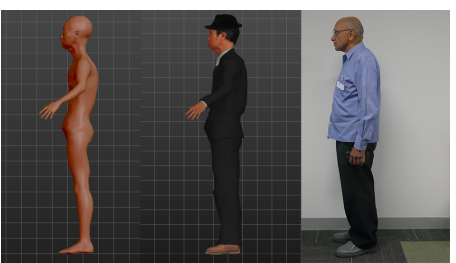

Figure 3: Keki's (male, 80) avatar with torso and hip manipulation.

\section{Introduction}

The study of online self-representations such as avatars has received a strong attention from researchers demonstrating how avatar's use can impact the experience in the virtual environment (VE). However, most of the studies have focused on younger populations [10]. Studies including older adults in this area have been mainly concerned in the healthcare or monitoring characteristic of an avatar representation e.g.[7]. Yet, not many studies have considered older adults' use of avatars in social contexts nor older adults as designers of their avatar self-representations. It is timely to learn about how older adults want to be portrayed online considering that two thirds of olde adults (65+) go online in the US and that an increasing percentage use social media or online games [1].

\section{Related Works}

There are many factors that influence the avatar design creation process. First, avatars are created for use in a particular context [5], hence an avatar for a gaming environment will be different from the one for a social context. The interface for designing the avatar also plays an important role in the design process emphasizing the use of some features over others [6] McArthur states for example that character creation interfaces constrain representations of gender and ethnicity [6]. There is not much study of the use of ageing traits in character creation interfaces which is vital to understand if these interfaces respond to older adults' customization preferences.

Each avatar design responds to personal selfrepresentation needs and while some people design avatars that reproduce their actual physical traits, others use avatars to explore an enhanced or alternative version of the self [5]. The selection of these features is not arbitrary as they can influence the behavior of the user in the VE; for example the use of a more attractive or taller avatar can make participants behave in a more friendly or confident way [10].

There is not much study of how older adults want to customize their avatars. Siriaraya and others studied adults (55+) suggesting that older adults enjoy using ageless avatars (avatars of a medium young age) as these allow participants to socialize with younger generations [8]. Other authors confirm that adults $(55+)$ prefer younger bodies, animal and object avatars when choosing a self-representation [4]. Throughout these few studies there are two major limitations. The first, is in relation to the age bracket as they target populations who were younger than 65 years, age that indicates the beginning of the older adulthood according to the World Health Organization [9]. The second limitation of these studies is that we do not know if the character creation interfaces display options that allow participants to customize their avatars adding ageing features.

\section{Research Question}

Based on the research gap, the main question of this study is: What role do virtual avatars play in supporting self-representation among older adults? This question will be explored through 3 sub questions; each corresponding a study described in the methods section: RQ1: How are older adults using selfrepresentations in online games?, RQ2: What are the representational characteristics of older adults when creating a humanoid avatar?;RQ3: How do avatarrelated attributes impact older adults' experience in a virtual reality environment? 


\section{Research Methods}

Study 1: Exploratory Study; Participants: 10 older adults social gamers $(65+)$

Data Collection: Observation of game sessions and indepth semi-structured interviews;

Data Analysis: Thematic Analysis

Study 2: Design Workshops Participants: 23 older adults (70+)

Data Collection: Field notes, semi-structured interviews, questionnaire, screenshots and avatars $3 \mathrm{~d}$ models; Data Analysis: Thematic Analysis

Study 3: Long-term User study;

Participants: 17 older adults (70+) who from Study 2 Data Collection: Observation of social virtual meetings, questionnaires, field notes, semi-structured interviews, screenshots, photographs; Data Analysis: Thematic Analysis

Table 1: Overview of research methods use in the three studies

\section{Method and Results}

The research uses a qualitative approach to generate a rich understanding of the design and use of avatars in relation to the ageing self. Table 1 provides an outline of the methods that will be used in this research. I aim to prioritize the voices of older adults to highlight their experiences over my interpretations. I start with an exploratory approach to understand current use of avatars and other type of self-representations of older adults' gamers. Then, I explore the design of avatars through two design sessions. The initial reflections of participants informed the following design session. Finally, a long-term user study aim to support participants' use and design' reiterations of avatars during multiple virtual social sessions.

Study1: Self-representations of Older Adults in Online Games

The study involved 10 older adults from 65 to 95 years old who play online games with other people. These findings suggest that despite the diversity of player self-representations, our participants portray elements of their past that they perceived they had lost such as youth or professional roles (see Figure 1, 2). These depictions may be a way of illustrating a nostalgic reflection of the lifetime. In addition self-

representations support older adults to embrace their actual older self by suggesting an acceptance of their role in society (see DIS 2018 full paper [3]).

Study 2: Older Adults Designing Avatars The understanding of the preferences of older adults when creating avatars was explored in two design sessions with the same group of older adults. The first session was conducted in a workshop setting with 23 participants $(70+)$ who had no previous knowledge about avatars. The findings of this study [2] show that the avatar designs of our participants share some similarities with avatar categories found in the literature. However, these avatar depictions created by our participants also suggest some specific visual negotiations in relation to the ageing body, including features such as age lines, stooped posture and reduced hair of head (see Figure 3 ). These findings contradict previous studies that suggested that older adults will prefer to create avatars different to their rea bodies $[4,5]$. Some of our participants also wanted to create enhanced version of themselves. In contrast to previous studies that suggest that these idealizations respond to Western ideals of beauty and prompt users to create avatars that look beautiful, thin and fashionable [5]; our participants idealized their depictions in relation to health, strength and youth. Finally, two of our participants created avatars as companions that represented persons from their past. To the best of understanding, this category has no similarity with the literature and may be responding to the social needs of later age. This study found that older adults have different interests when creating their avatars and that the graphical interface they used limited the representation of ageing features.

We followed up this study with an individual avatar design session using commercial software (Character Creator) with better photorealistic and ageing customization options. We asked participants to create a replica of themselves (using actual pictures of their faces) and a cartoon avatar. While this data is still under analysis, the picture-based avatar (see Figure 4) supported a deeper exploration of the depiction of ageing bodies and the cartoon avatar triggered a more playful exploration of self (see Figure 5). 


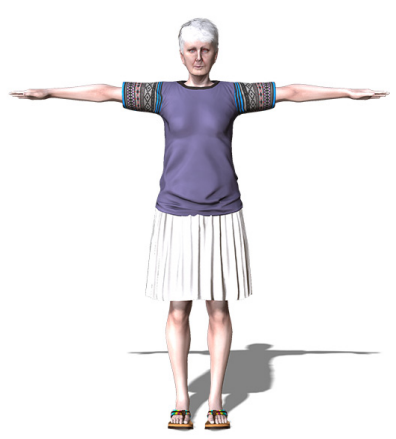

Figure 4: Holly's (female, 81) picture base avatar.

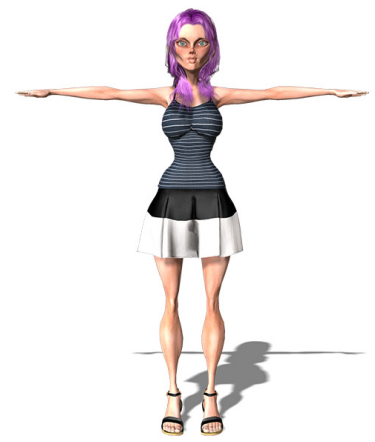

Figure 5: Holly's (female, 81) cartoon avatar.

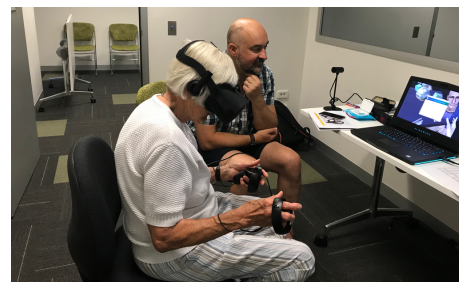

Figure 6: Holly (female, 81) using her avatar in a first person view.
Study 3: Older Adults using avatars for socializing in VE Participants who partook in the previous study designing avatars were then invited to join a series of virtual social meetings. To participate in each virtual session, they first have to choose their self-

representation between the picture base and cartoon avatars they previously designed. The use of a head mounted display is supporting a first person perspective of their avatar' virtual body (see Figure 6). The virtual social meetings will group 3 participants and a facilitator sited around a table where they will prompted to discuss life stories. My aim in this study is to compare the use of these two avatars (picture-based and cartoon avatar) to understand how they impact older adults' experience when socializing with their peers in the VE.

This study brings interesting challenges such as how to evaluate the impact of avatar's preferences in the social interactions in the VE. Finally, considering that participants would also have opportunities to reiterate their avatar designs alongside the virtual reality meetings, I should be able to have an understanding of the social implications of their avatar designs reiterations in the long-term use.

\section{Acknowledgements}

Assistance provided by Dr. Jenny Waycott, Prof. Frank Vetere, and Dr. Steven was greatly appreciated.

\section{References}

1. Monica Anderson and Andrew Perrin. 2017. Tech adoption climbs among older adults. Pew Research Center, May: 1-22. Retrieved from http://www.pewinternet.org/2017/05/17/technolog y-use-among-seniors/
2. Romina Carrasco, Steven Baker, Jenny Waycott, and Frank Vetere. Negotiating stereotypes of older adults through avatars. In Proc. OZCHI 2017, ACM Press (2017), 218-227.

3. Romina Carrasco, Jenny Waycott, Steven Baker, and Frank Vetere. Designing the Lost Self : Older Adults' Self - representations in Online Games. In Proc. DIS 2018, ACM Press (2018).

4. Wei Lun Cheong, Younbo Jung, and Yin-Leng Theng. Avatar: A Virtual Face for the Elderly. In Proc. VRCAI 2011, ACM Press (2011), 491-498.

5. Nicolas Ducheneaut, Ming-Hui "Don" Wen, Nicholas Yee, and Greg Wadley. Body and Mind: A Study of Avatar Personalization in Three Virtual Worlds. In Proc. CHI 2009, ACM Press (2009), 1151-1160.

6. Victoria Mcarthur, Robert J Teather, and Jennifer Jenson. The Avatar Affordances Framework: Mapping Affordances and Design Trends in Character Creation Interfaces. In Proc. CHI PLAY 2015, ACM Press (2015), 231-240.

7. Matti Pouke and Jonna Häkkilä. 2013. Elderly healthcare monitoring using an avatar-based 3D virtual environment. International Journal of Environmental Research and Public Health 10, 12: 7283-7298. https://doi.org/10.3390/ijerph10127283

8. Panote Siriaraya, Chee Siang Ang, and Ania Bobrowicz. 2014. Exploring the potential of virtual worlds in engaging older people and supporting healthy aging. Behaviour \& Information Technology 33, 3: 282-293.

https://doi.org/10.1080/0144929X.2012.691552

9. World Health Organization. 2015. World report on Ageing And Health. Luxembourg. https://doi.org/10.1007/s13398-014-0173-7.2

10. Nick Yee. 2014. Proteus paradox: how online games and virtual worlds change us, and how they don't. Yale University Press. 


\section{University Library}

\section{- M M N E R VA A gateway to Melbourne's research publications}

Minerva Access is the Institutional Repository of The University of Melbourne

Author/s:

Carrasco Zuffi, R

Title:

Older Adults Designing Avatars for Socializing

Date:

2018

Citation:

Carrasco Zuffi, R. (2018). Older Adults Designing Avatars for Socializing. Proceedings of the 19th International ACM SIGACCESS Conference on Computers and Accessibility - DIS '18, pp.379-382. ACM Press. https://doi.org/10.1145/3197391.3205387.

Persistent Link:

http://hdl.handle.net/11343/242028 